\title{
APPROXIMATIONS FOR VON NEUMANN AND RÉNYI ENTROPIES OF GRAPHS USING THE EULER-MACLAURIN FORMULA*
}

\author{
NATÁLIA BEBIANO ${ }^{\dagger}$, SUSANA FURTADO ${ }^{\ddagger}$, JOÃO DA PROVIDÊNCIA§, WEI-RU XUף, AND \\ JOÃO P. DA PROVIDÊNCIA\|
}

\begin{abstract}
There have been many attempts of understanding graph structures by investigating graph entropies. In this article we investigate approximations for von Neumann and Rényi- $\alpha$ entropies of paths and rings, using the Euler-Maclaurin summation formula. For $\alpha$ an integer, the approximations become exact, and, in general, the obtained estimates have a remarkable degree of accuracy.
\end{abstract}

Key words. entropy, graphs, Laplacian matrix, Euler-Maclaurin formula

AMS subject classifications. 05C50, 81P45, 91A17

1. Introduction. The Euler-Maclaurin (E-M) formula is an important tool in numerical analysis and one of the most remarkable formulas in mathematics. It estimates a sum $\sum_{k=0}^{n} g(k)$ through the integral $\int_{0}^{n} g(t) \mathrm{d} t$ with an error term involving Bernoulli numbers [13]. One form of the E-M formula states

$$
\begin{aligned}
\sum_{k=0}^{n-1} g(k)= & \int_{0}^{n} g(t) \mathrm{d} t-\frac{1}{2}(g(n)-g(0)) \\
& +\frac{1}{12}\left(g^{\prime}(n)-g^{\prime}(0)\right)-\frac{1}{2} \int_{0}^{n} B_{2}(\{t\}) g^{\prime \prime}(t) \mathrm{d} t,
\end{aligned}
$$

where $k$ is a nonnegative integer, $B_{2}(x)=x^{2}-x+1 / 6$ is the second Bernoulli polynomial, and $\{t\}$ denotes the fractional part of $t$. The condition imposed on the real function $g$ is that it should have a continuous second derivative for $t \in(0, n)$. If $g$ has a continuous second derivative for $t \in[0, n]$ and a continuous third derivative for $t \in(0, n)$, then the following form holds (see [8]):

$$
\begin{aligned}
\sum_{k=0}^{n-1} g(k)= & \int_{0}^{n} g(t) \mathrm{d} t-\frac{1}{2}(g(n)-g(0)) \\
& +\frac{1}{12}\left(g^{\prime}(n)-g^{\prime}(0)\right)+\frac{1}{6} \int_{0}^{n} B_{3}(\{t\}) g^{\prime \prime \prime}(t) \mathrm{d} t
\end{aligned}
$$

where $B_{3}(x)=x^{3}-3 x^{2} / 2+x / 2$ is the third Bernoulli polynomial.

*Received April 13, 2018. Accepted May 11, 2018. Published online on June 28, 2018. Recommended by L. Reichel.

${ }^{\dagger}$ CMUC, University of Coimbra, Department of Mathematics, P 3001-454 Coimbra, Portugal (bebiano@mat.uc.pt).

${ }^{\ddagger}$ CEAFEL and Faculty of Economy, University of Oporto, 4200-464 Oporto, Portugal (sb $f @ f e p$. up.pt).

${ }^{\S}$ CFisUC, Department of Physics, University of Coimbra, P 3004-516 Coimbra, Portugal (providencia@teor.fis.uc.pt).

ISchool of Mathematical Sciences, Shanghai Key Laboratory of Pure Mathematics and Mathematical Practice, East China Normal University, Shanghai 200241, P. R. China (weiruxuefoxmail.com, weiruxu@mat.uc.pt). "Department of Physics, Univ. of Beira Interior, P-6201-001 Covilhã, Portugal
(joaodaprovidencia@daad-alumni.de). 


\section{ETNA}

Kent State University and

Johann Radon Institute (RICAM)

Under the weaker hypothesis that $g^{\prime}(0)$ or $g^{\prime}(n)$ do not exist, i.e., $g^{\prime}(t)$ exists only for $t \in(0, n)$, instead of (1.1) the following holds:

$$
\sum_{k=0}^{n-1} g(k)=\int_{0}^{n} g(t) \mathrm{d} t-\frac{1}{2}(g(n)-g(0))+\int_{0}^{n} B_{1}(\{t\}) g^{\prime}(t) \mathrm{d} t,
$$

where $B_{1}(x)=x-1 / 2$ is the first Bernoulli polynomial.

In this paper we discuss the application of the E-M formula in graph entropy. The E-M formula is an important tool for numerical integration and numerical summation [14]. Mathematica also uses this famous formula [14, p. 917]. Interestingly, neither Euler nor Maclaurin presented this formula with remainder. The first one to do so was Poisson in 1830. Since then, it has been derived in different ways (see [1] and [8] for elementary derivations).

The notion of entropy is due to Rudolf Clausius (1850) and is connected with his famous theorem which generalizes the equally famous Carnot Theorem on the efficiency of thermal machines. The concept has gained many applications in several research areas such as statistical mechanics, information theory, etc. Recently, there have been many attempts of understanding graph structures by investigating graph entropies (see [3, 4, 12, 15] and references therein).

Let $G$ be an undirected graph with $n$ vertices and at least one edge, and let $L(G)$ be the combinatorial Laplacian matrix of $G$, that is, $L(G)=D(G)-A(G)$, where $D(G)$ is the diagonal matrix whose $(i, i)$-th entry is the degree of the vertex $i$ and $A(G)$ is the adjacency matrix of $G$ [6]. Note that each row (and column) sum of $L(G)$ is 0 , and so $L(G)$ is singular. Normalizing this matrix by its trace, we get

$$
\rho(G)=\frac{1}{\operatorname{Tr} L(G)} L(G),
$$

called the density matrix of $G$. By the Gershgorin Theorem, all eigenvalues of $\rho(G)$ are nonnegative [7]. Thus, $G$ can be seen as a quantum state since $\rho(G)$ is a Hermitian positive semidefinite matrix with unit trace. Therefore, it is natural to investigate the information content of the graph as a quantum state [10].

Let $\lambda_{1}, \ldots, \lambda_{n}$ be the eigenvalues of $\rho(G)$. Note that

$$
\lambda_{1}+\cdots+\lambda_{n}=1 .
$$

The von Neumann entropy of $G$ is defined as

$$
S(G)=-\sum_{i=1}^{n} \lambda_{i} \log _{2} \lambda_{i}
$$

From now on, we will use the natural logarithm in the definitions of the entropy. (The two definitions, using $\log _{2}$ or $\log$, are equivalent up to a positive constant.) We make the convention that $0 \log 0=0$. We will use the following notations for a graph on $n$ vertices: $K_{1, n-1}$ denotes the star graph, $P_{n}$ denotes the path, $C_{n}$ denotes the ring, and $K_{n}$ denotes the complete graph.

It is well known that the Laplacian spectrum of the star graph is

$$
\sigma\left(L\left(K_{1, n-1}\right)\right)=\{0, n, 1, \ldots, 1\},
$$

where the eigenvalue 1 has multiplicity $n-2$. Thus, we have

$$
S\left(K_{1, n-1}\right)=\log (2 n-2)-\frac{n}{2 n-2} \log (n),
$$


and

$$
\lim _{n \rightarrow \infty} \frac{S\left(K_{1, n-1}\right)}{\log (n-1)}=\frac{1}{2}
$$

In [3, Conjecture 1.3], it has been conjectured that

CONJECTURE 1.1. For all connected graphs $G$ on $n$ vertices,

$$
S\left(K_{1, n-1}\right) \leq S(G) .
$$

The conjecture was proved for almost all graphs with $n$ vertices [3, Theorem 2.3]. In the same article the following conjecture was also formulated:

CONJECTURE 1.2. For any tree $T$ on $n$ vertices,

$$
S(T) \leq S\left(P_{n}\right) .
$$

Let $G$ be a graph with at least one edge. Consider the density matrix $\rho(G)$. For $\alpha \in(0,1) \cup$ $(1, \infty)$ fixed, the Rényi- $\alpha$ entropy of $G[11]$ is defined as

$$
H_{\alpha}(G)=\frac{1}{1-\alpha} \log \sum_{i=1}^{n} \lambda_{i}^{\alpha}
$$

and is also denoted as $H_{\alpha}(\rho(G))$. For a fixed graph $G$, the Rényi- $\alpha$ entropy $H_{\alpha}(G)$ is a monotonically decreasing function of $\alpha$ [3],

$$
H_{\alpha}(G) \leq H_{\alpha^{\prime}}(G) \quad \text { for } \alpha>\alpha^{\prime} .
$$

It was proved in [3, Proposition 3.1] that $H_{\alpha}(\lambda)$, for $\alpha>1$ and $n \geq 1$, when viewed as a function of a probability distribution, $\lambda=\left(\lambda_{1}, \ldots, \lambda_{n}\right)$

1. is minimized by the distribution $\lambda_{0}=(1,0, \ldots, 0)$, and this is the only probability distribution (up to a permutation of the entries) that does so;

2. is maximized by the constant distribution $\lambda_{c}=(1 / n, \ldots, 1 / n)$. We have

$$
H_{\alpha}\left(K_{1, n-1}\right)=(1-\alpha)^{-1} \log \left(\left(n^{\alpha}+n-2\right)(2 n-2)^{-\alpha}\right) .
$$

In [3, Conjecture 3.3], the following conjecture has been formulated.

CONJECTURE 1.3. For $\alpha>1$ and any connected graph $G$ on $n$ vertices,

$$
H_{\alpha}\left(K_{1, n-1}\right) \leq H_{\alpha}(G)
$$

Note that, as

$$
\lim _{\alpha \rightarrow 1^{+}} H_{\alpha}(G)=S(G),
$$

the veracity of Conjecture 1.3 implies that Conjecture 1.1 has a positive answer.

This article is organized as follows: In Section 2 some useful background is presented. In Section 3, estimates of the Rényi- $\alpha$ entropy of paths and rings on $n$ vertices are obtained for $\alpha \in(1, \infty)$. The obtained approximations are shown to be exact in the case of $\alpha$ being an integer. In Section 4, approximations for the von Neumann entropy are given. The key tool for obtaining these estimates is the E-M formula. In Section 5, some final remarks are presented. 
2. Preliminary results. Given a path $P_{n}$, up to a permutation similarity, $L\left(P_{n}\right)$ is the tridiagonal matrix

$$
L\left(P_{n}\right)=\left[\begin{array}{ccccc}
1 & -1 & 0 & \cdots & 0 \\
-1 & 2 & -1 & & \\
0 & -1 & \ddots & \ddots & \vdots \\
\vdots & & \ddots & 2 & -1 \\
0 & & \cdots & -1 & 1
\end{array}\right] .
$$

The eigenvalues of $L\left(P_{n}\right)$ are well known in the literature and can be readily obtained [6].

Lemma 2.1. Let $P_{n}$ be a path on $n$ vertices. Then the eigenvalues of $L\left(P_{n}\right)$ are

$$
\beta_{k}=2+2 \cos (k \pi / n), \quad k=1, \ldots, n .
$$

The Laplacian matrix of the ring $C_{n}$ is the circulant matrix

$$
L\left(C_{n}\right)=\left[\begin{array}{ccccc}
2 & -1 & 0 & & -1 \\
-1 & 2 & -1 & & \\
0 & -1 & \ddots & \ddots & \\
& & \ddots & 2 & -1 \\
-1 & 0 & & -1 & 2
\end{array}\right]
$$

LEMMA 2.2 ([4]). The eigenvalues of $L\left(C_{n}\right)$ are

$$
\beta_{k}=2-2 \cos (2 \pi k / n), \quad k=1,2, \ldots, n .
$$

Proposition 2.3. The Rényi-2 entropies of $P_{n}$ and $C_{n}$ are, respectively,

$$
H_{2}\left(P_{n}\right)=2 \log (2 n-2)-\log (6 n-8) \quad \text { and } \quad H_{2}\left(C_{n}\right)=2 \log (2 n)-\log (6 n) .
$$

Proof. Observing that $\operatorname{Tr} P_{n}=2 n-2, \operatorname{Tr} C_{n}=2 n$, by easy computations, we get

$$
\sum_{k=1}^{n}\left(2+2 \cos \frac{k \pi}{n}\right)^{2}=6 n-8, \quad \text { and } \quad \sum_{k=1}^{n}\left(2-2 \cos \frac{2 k \pi}{n}\right)^{2}=6 n,
$$

and the result follows.

\section{On the Rényi- $\alpha$ entropy of paths and rings.}

3.1. Estimates for paths. The main results in this section are Theorems 3.5 and 3.6. To prove them, some auxiliary lemmas are needed. We start by presenting them. Throughout the article, the following form of the E-M formula is used:

LEMMA 3.1. Let $n$ be a positive integer, and let $f$ be a real function of class $C^{2}$ in $[0,1]$. Then

$$
\sum_{k=1}^{n} f(k / n)=n \int_{0}^{1} f(x) \mathrm{d} x+\frac{1}{2}(f(1)-f(0))+\frac{1}{12 n}\left(f^{\prime}(1)-f^{\prime}(0)\right)+R_{n},
$$

with

$$
R_{n}=\frac{1}{6 n^{2}} \int_{0}^{1} B_{3}(\{n x\}) f^{\prime \prime \prime}(x) \mathrm{d} x,
$$

$B_{3}(x)=x^{3}-3 x^{2} / 2+x / 2$ the third Bernoulli polynomial, and $\{x\}$ the fractional part of $x$. 
Proof. By the E-M formula (1.2), we have

$$
\begin{aligned}
\sum_{k=1}^{n} f(k / n)= & f(n / n)-f(0 / n)+\sum_{k=0}^{n-1} f(k / n) \\
= & f(n / n)-f(0 / n)+\int_{0}^{n} f(t / n) \mathrm{d} t-\frac{1}{2}(f(n / n)-f(0 / n)) \\
& \quad+\left.\frac{1}{12} \frac{\mathrm{d} f(t / n)}{\mathrm{d} t}\right|_{t=0} ^{t=n}+\frac{1}{6} \int_{0}^{n} B_{3}(\{t\}) \frac{\mathrm{d}^{3}(t / n)}{\mathrm{d} t^{3}} \mathrm{~d} t \\
= & \int_{0}^{n} f(t / n) \mathrm{d} t+\frac{1}{2}(f(n / n)-f(0 / n)) \\
& \quad+\left.\frac{1}{12} \frac{\mathrm{d} f(t / n)}{\mathrm{d} t}\right|_{t=0} ^{t=n}+\frac{1}{6} \int_{0}^{n} B_{3}(\{t\}) \frac{\mathrm{d}^{3} f(t / n)}{\mathrm{d} t^{3}} \mathrm{~d} t
\end{aligned}
$$

Changing variables $x=t / n$, the result follows.

LEMMA 3.2. Let $f: \mathbb{R} \rightarrow \mathbb{R}$

$$
f(x):=(2+2 \cos (\pi x))^{\alpha}, \quad \alpha \in \mathbb{R} .
$$

Then for $R_{n}$ in (3.2) and $\alpha>1$

$$
\lim _{n \rightarrow \infty} n R_{n}=0
$$

Proof. Notice that

$$
\begin{aligned}
& f^{\prime \prime}(x)=-\frac{\alpha \pi^{2}}{2}(2+2 \cos (\pi x))^{\alpha}(1-\alpha+\alpha \cos (\pi x)) \sec ^{2}(\pi x / 2) \\
& f^{\prime \prime \prime}(x)=\frac{\alpha \pi^{3}}{2}(2+2 \cos (\pi x))^{\alpha}\left(-1+3 \alpha-\alpha^{2}+\alpha^{2} \cos (\pi x)\right) \sec ^{2}(\pi x / 2) \tan (\pi x / 2)
\end{aligned}
$$

and that $f^{\prime \prime \prime}\left(x_{0}\right)=0$ for

$$
x_{0}=\frac{1}{\pi} \arccos \left(\left(1-3 \alpha+\alpha^{2}\right) / \alpha^{2}\right),
$$

where $f^{\prime \prime \prime}(x)$ changes sign. By easy computations and having (1.2) in mind, we find for $\alpha>1$,

$$
\begin{aligned}
\int_{0}^{1}\left|f^{\prime \prime \prime}(x)\right| \mathrm{d} x & =f^{\prime \prime}(0)+f^{\prime \prime}(1)-2 f^{\prime \prime}\left(x_{0}\right) \\
& =\frac{\alpha\left(-4^{\alpha}+\left(4^{\alpha}+4\left(4+2 / \alpha^{2}-6 / \alpha\right)^{\alpha}\right) \alpha\right) \pi^{2}}{2(-1+\alpha)}=: d_{\alpha} .
\end{aligned}
$$

Having in mind that

$$
\left|B_{3}(x)\right| \leq \frac{1}{12 \sqrt{3}}
$$

we get

$$
\left|R_{n}\right| \leq \frac{d_{\alpha}}{72 \sqrt{3} n^{2}}
$$

and the result follows. 
Throughout, we use the following formula as given in Mathematica:

$$
\int_{0}^{1}(2+2 \cos (2 \pi x))^{\alpha} \mathrm{d} x=\frac{4^{\alpha} \Gamma(1 / 2+\alpha)}{\sqrt{\pi} \Gamma(1+\alpha)}=: c_{\alpha},
$$

where $\alpha>-1 / 2$ and $\Gamma$ is the well-known Gamma function.

LeMma 3.3. For $\alpha>1$, we have

$$
\lim _{n \rightarrow \infty} n\left(\sum_{k=1}^{n}(2+2 \cos (k \pi / n))^{\alpha}-\frac{4^{\alpha} \Gamma(1 / 2+\alpha)}{\sqrt{\pi} \Gamma(1+\alpha)} n+\frac{4^{\alpha}}{2}\right)=0 .
$$

Proof. For $f(x):=(2+2 \cos (\pi x))^{\alpha}$, we have $f(1)=0, f(0)=4^{\alpha}, f^{\prime}(1)=0$, $f^{\prime}(0)=0$. This function is of class $C^{2}$ in the interval $[0,1]$. By Lemma 3.1, we get

$$
\begin{aligned}
\sum_{k=1}^{n}(2+2 \cos \pi k / n)^{\alpha} & =n \int_{0}^{1} f(x) \mathrm{d} x+\frac{1}{2}(f(1)-f(0))+\frac{1}{12 n}\left(f^{\prime}(1)-f^{\prime}(0)\right)+R_{n} \\
& =n \frac{4^{\alpha} \Gamma(1 / 2+\alpha)}{\sqrt{\pi} \Gamma(1+\alpha)}-\frac{4^{\alpha}}{2}+R_{n},
\end{aligned}
$$

where $n R_{n} \rightarrow 0$ as $n \rightarrow \infty$, by Lemma 3.2.

In Table 3.1 we compare, for $n=40$, the sum $\sum_{k=0}^{n} \beta_{k}^{\alpha}$, where $\beta_{1}, \ldots, \beta_{n}$ are the eigenvalues of $L\left(P_{n}\right)$, its approximation $c_{\alpha} n-4^{\alpha} / 2$, and $R_{n}$. The vanishing values of $R_{n}$ suggest that, for $\alpha$ a positive integer, $R_{n}=0$.

\begin{tabular}{clcc}
\multicolumn{5}{c}{ TABLE 3.1 } \\
Comparing $\sum_{k=0}^{n} \beta_{k}, c_{\alpha} n-4^{\alpha} / 2$, and $R_{n}$, for $n=40$. \\
$\alpha$ & $\sum_{k=0}^{n} \beta_{k}^{\alpha}$ & $c_{\alpha} n-4^{\alpha} / 2$ & $R_{n}$ \\
\hline $3 / 2$ & 131.812 & 131.812 & $4.03876 \times 10^{-6}$ \\
2 & 232 & 232 & 0 \\
$5 / 2$ & 418.599 & 418.599 & $-1.1875 \times 10^{-8}$ \\
3 & 768 & 768 & 0 \\
$7 / 2$ & 1426.05 & 1426.05 & $7.7307 \times 10^{-11}$ \\
4 & 2672 & 2672 & 0 \\
$9 / 2$ & 5041.97 & 5041.97 & $9.09495 \times 10^{-13}$ \\
5 & 9568 & 9568 & 0
\end{tabular}

It is interesting to consider the case when $\alpha$ is an integer. For $\alpha=m \in \mathbb{Z}^{+}$, we may write

$$
\begin{aligned}
\frac{4^{\alpha} \Gamma(1 / 2+\alpha)}{\sqrt{\pi} \Gamma(1+\alpha)} & =\frac{4^{m} \Gamma(1 / 2+m)}{\sqrt{\pi} \Gamma(1+m)}=\frac{4^{m}(m-1 / 2)(m-3 / 2) \cdots 1 / 2}{m !} \\
& =\frac{2^{m}(2 m-1) ! !}{m !}
\end{aligned}
$$

where $(2 m-1) ! !=(2 m-1)(2 m-3) \cdots 1$.

Lemma 3.4. For $m, n \in \mathbb{Z}^{+}$,

$$
\sum_{l=1}^{n}(2+2 \cos (\pi l / n))^{m}=n 2^{m} \frac{(2 m-1) ! !}{m !}-\frac{4^{m}}{2} .
$$


Proof. For $t \in \mathbb{R}$ and $p, k \in \mathbb{Z}^{+}$, by the binomial theorem, the following identity holds:

$$
(2+2 \cos (t))^{m}=2^{m} \sum_{p=0}^{m} \cos ^{p}(t)\left(\begin{array}{c}
m \\
p
\end{array}\right), \quad m \in \mathbb{Z}^{+} .
$$

Considering $\left(\mathrm{e}^{i t}+\mathrm{e}^{-i t}\right) / 2=\cos (t)$, we easily find, again by the binomial theorem, that

$$
\left(\frac{\mathrm{e}^{i t}+\mathrm{e}^{-i t}}{2}\right)^{p}=\frac{1}{2^{p}} \sum_{k=0}^{p}\left(\begin{array}{l}
p \\
k
\end{array}\right) \mathrm{e}^{2 i k t-i t p}=\frac{1}{2^{p}} \sum_{k=1}^{p}\left(\begin{array}{l}
p \\
k
\end{array}\right) \cos (2 k t-t p)
$$

because $\left(\begin{array}{l}p \\ k\end{array}\right)=\left(\begin{array}{c}p \\ p-k\end{array}\right)$. Hence,

$$
\cos ^{p}(t)=\frac{1}{2^{p}} \sum_{k=0}^{p} \cos ((2 k-p) t)\left(\begin{array}{l}
p \\
k
\end{array}\right) .
$$

As the sum of the $n$ roots of unity is zero,

$$
\sum_{k=1}^{n} \mathrm{e}^{2 i k s \pi / n}=0, \quad 0 \neq s \in \mathbb{Z}
$$

for $r=2 s, 0 \neq s \in \mathbb{Z}$, we have

$$
\sum_{k=1}^{n} \cos (\pi r k / n)=0
$$

For $r=2 s+1, s \in \mathbb{Z}$, it can be easily seen that

$$
\sum_{k=1}^{n} \cos (\pi r k / n)=-1 .
$$

Thus,

$$
\sum_{l=1}^{n}(2+2 \cos (\pi l / n))^{m}=n 2^{m} \sum_{q=0}^{[m / 2]} \frac{m !}{2^{2 q}(m-2 q) ! q ! q !}-2^{m} \sum_{q=0}^{q_{\max }}\left(\begin{array}{c}
m \\
2 q+1
\end{array}\right),
$$

where $q_{\max }=m / 2-1$ if $m$ is even and $q_{\max }=(m-1) / 2$ if $m$ is odd.

The following identity holds (see [2]):

$$
\sum_{q=0}^{[m / 2]} \frac{m !}{2^{2 q}(m-2 q) ! q ! q !}=\frac{(2 m-1) ! !}{m !} .
$$

We easily conclude that

$$
2^{m} \sum_{q=0}^{q_{\max }}\left(\begin{array}{c}
m \\
2 q+1
\end{array}\right)=\frac{4^{m}}{2}
$$

and the result follows.

THEOREM 3.5. For $\alpha$ an integer and $c_{\alpha}$ in (3.3), the E-M approximation

$$
n c_{\alpha}-\frac{4^{\alpha}}{20}
$$


to the sum

$$
\sum_{l=1}^{n}(2+2 \cos (\pi l / n))^{\alpha}
$$

is exact.

Proof. This is a simple consequence of Lemma 3.4.

We recall that

$$
H_{\alpha}\left(P_{n}\right)=\frac{1}{1-\alpha}\left(\log \sum_{k=1}^{n} \beta_{k}^{\alpha}-\alpha \log (2 n-2)\right),
$$

where the $\beta_{k}$ are the eigenvalues of $L\left(P_{n}\right)$ in (2.1). We use the notation

$$
\widetilde{H}_{\alpha}\left(P_{n}\right)=\frac{1}{1-\alpha}\left(\log \left(\frac{4^{\alpha} \Gamma(1 / 2+\alpha)}{\sqrt{\pi} \Gamma(1+\alpha)} n-\frac{4^{\alpha}}{2}\right)-\alpha \log (2 n-2)\right) .
$$

THEOREM 3.6. For $\alpha>1$ and $\widetilde{H}_{\alpha}\left(P_{n}\right)$ in (3.5), we have

$$
\lim _{n \rightarrow \infty} n\left(H_{\alpha}\left(P_{n}\right)-\widetilde{H}_{\alpha}\left(P_{n}\right)\right)=0 .
$$

Further, it holds that

$$
\lim _{n \rightarrow \infty} \frac{H_{\alpha}\left(P_{n}\right)}{\log (n-1)}=1 .
$$

Proof. Let $\beta_{1}, \ldots, \beta_{n}$ be the eigenvalues of $L\left(P_{n}\right)$. We may write

$$
\begin{aligned}
H_{\alpha}\left(P_{n}\right) & =\frac{1}{(1-\alpha)}\left(\log \sum_{i=1}^{n} \beta_{i}^{\alpha}-\alpha \log (2 n-2)\right) \\
& =\frac{1}{1-\alpha}\left(\log \left(\frac{4^{\alpha} \Gamma(1 / 2+\alpha)}{\sqrt{\pi} \Gamma(1+\alpha)} n-\frac{4^{\alpha}}{2}+R_{n}\right)-\alpha \log (2 n-2)\right)
\end{aligned}
$$

so that for $c_{\alpha}$ in (3.3) we find

$$
\begin{aligned}
n\left(H_{\alpha}\left(P_{n}\right)-\widetilde{H}_{\alpha}\left(P_{n}\right)\right) & =\frac{1}{1-\alpha}\left(\log \left(c_{\alpha} n-\frac{4^{\alpha}}{2}+R_{n}\right)^{n}-\log \left(c_{\alpha} n-\frac{4^{\alpha}}{2}\right)^{n}\right) \\
& =\frac{1}{1-\alpha}\left(\log \left(1+\frac{R_{n}}{c_{\alpha} n-4^{\alpha} / 2}\right)^{n}\right) .
\end{aligned}
$$

We have

$$
\lim _{n \rightarrow \infty} \log \left(1+\frac{R_{n}}{c_{\alpha} n-4^{\alpha} / 2}\right)^{n}=\log \lim _{n \rightarrow \infty}\left(1+\frac{R_{n}}{n\left(c_{\alpha}-4^{\alpha} /(2 n)\right)}\right)^{n}=0
$$

because $\lim _{n \rightarrow \infty} \frac{R_{n}}{c_{\alpha}-4^{\alpha} /(2 n)}=0$. It follows that

$$
\lim _{n \rightarrow \infty} n\left(H_{\alpha}\left(P_{n}\right)-\widetilde{H}_{\alpha}\left(P_{n}\right)\right)=0 .
$$

We may also write

$$
H_{\alpha}\left(P_{n}\right)=\log (n-1)+\frac{\log \left(c_{\alpha}+\left(c_{\alpha}-4^{\alpha} / 2+R_{n}\right) /(n-1)\right)-\alpha \log 2}{1-\alpha}
$$

so that

$$
\frac{H_{\alpha}\left(P_{n}\right)}{\log (n-1)}=1+\frac{\log \left(c_{\alpha}+\left(c_{\alpha}-4^{\alpha} / 2+R_{n}\right) /(n-1)\right)-\alpha \log 2}{(1-\alpha) \log (n-1)} .
$$

Then, the last statement follows. 


\section{ETNA}

Kent State University and

Johann Radon Institute (RICAM)

\begin{tabular}{rcccc}
\multicolumn{5}{c}{ Comparing $H_{3 / 2}\left(P_{n}\right), \widetilde{H}_{3 / 2}^{\text {TABLE }} 3.2$} \\
\multicolumn{7}{c}{$\left(P_{n}\right), H_{3 / 2}\left(K_{1, n-1}\right)$, and $n R_{n}}$. \\
$n$ & $H_{3 / 2}\left(P_{n}\right)$ & $\widetilde{H}_{3 / 2}\left(P_{n}\right)$ & $H_{3 / 2}\left(K_{1, n-1}\right)$ & \multicolumn{1}{c}{$n R_{n}$} \\
\hline 5 & 1.11171414 & 1.11204036 & 0.934611644 & 0.0105839 \\
10 & 1.87183531 & 1.87185266 & 1.31230703 & 0.00259912 \\
20 & 2.59792755 & 2.59792856 & 1.55884276 & 0.000646914 \\
40 & 3.30736978 & 3.30736984 & 1.72360220 & 0.000161550 \\
80 & 4.00861581 & 4.00861582 & 1.83477278 & 0.0000403765 \\
160 & 4.70579976 & 4.70579976 & 1.91029025 & 0.0000100935
\end{tabular}

3.1.1. Numerical experiments. In Table 3.2 we compare the Rényi- $3 / 2$ entropy of the path $P_{n}$ with the approximate result in Theorem 3.6, denoted by $\widetilde{H}_{3 / 2}\left(P_{n}\right)$, and with the Rényi-3/2 entropy of $K_{1, n-1}$ using equations (3.4), (3.5), and (1.5). The values of $n R_{n}$ are also presented, suggesting that $n R_{n}$ behaves like $1 / n^{2}$ so that $R_{n}$ behaves like $1 / n^{3}$.

Notice that (3.5), which has been derived having (1.1) in mind, remains valid also if $\alpha<1$. In this case, we use (1.3), the only difference is the so obtained $R_{n}$ because in the actual application of the formula it turns out that $g^{\prime}(n)=g^{\prime}(0)=0$.

In Table 3.3 we compare the Rényi- $1 / 2$ entropy of the path $P_{n}$ with $\widetilde{H}_{1 / 2}\left(P_{n}\right)$ and with the Rényi-1/2 entropy of $K_{1, n-1}$ using equations (3.4), (3.5), and (1.5). The values of $n R_{n}$ are also presented, suggesting that $n R_{n}$ remains almost constant so that $R_{n}$ behaves like $1 / n$.

\begin{tabular}{rcccc}
\multicolumn{6}{c}{ TABLE 3.3} \\
$n$ & $H_{1 / 2}\left(P_{n}\right)$ & $\widetilde{H}_{1 / 2}\left(P_{n}\right)$ & $H_{1 / 2}\left(K_{1, n-1}\right)$ & $n R_{n}$ \\
\hline 5 & 1.26115463 & 1.28079766 & 1.23170012 & -0.262231 \\
10 & 2.02986628 & 2.03433596 & 1.93470830 & -0.261907 \\
20 & 2.75581329 & 2.75688380 & 2.58696612 & -0.261826 \\
40 & 3.46425628 & 3.46451847 & 3.22636881 & -0.261806 \\
80 & 4.16479032 & 4.16485521 & 3.86793969 & -0.261801 \\
160 & 4.86156740 & 4.86158355 & 4.51716754 & -0.261800
\end{tabular}

\begin{tabular}{rcccc}
\multicolumn{5}{c}{ TABLE 3.4} \\
\multicolumn{5}{c}{ Comparing $H_{1 / 4}\left(P_{n}\right), \widetilde{H}_{1 / 4}\left(P_{n}\right), H_{1 / 4}\left(K_{1, n-1}\right)$, and $\sqrt{n} R_{n}}$. \\
$n$ & $H_{1 / 4}\left(P_{n}\right)$ & $\widetilde{H}_{1 / 4}\left(P_{n}\right)$ & $H_{1 / 4}\left(K_{1, n-1}\right)$ & $\sqrt{n} R_{n}$ \\
\hline 5 & 1.31866798 & 1.36641122 & 1.31091050 & -0.368592936 \\
10 & 2.10176931 & 2.11727319 & 2.07676080 & -0.368499782 \\
20 & 2.83308794 & 2.83836306 & 2.78940862 & -0.368476500 \\
40 & 3.54342184 & 3.54525341 & 3.48332238 & -0.368470680 \\
80 & 4.24457358 & 4.24521550 & 4.17158073 & -0.368469225 \\
160 & 4.94153005 & 4.94175603 & 4.85912326 & -0.368468861
\end{tabular}

In Table 3.4 we compare the Rényi-1/4 entropy of the path $P_{n}$ with $\widetilde{H}_{1 / 4}\left(P_{n}\right)$ and with the Rényi-1/4 entropy of $K_{1, n-1}$ using (3.4), (3.5), and (1.5). The values of $\sqrt{n} R_{n}$ are also presented, suggesting that $\sqrt{n} R_{n}$ remains almost constant so that $R_{n}$ behaves like $1 / \sqrt{n}$. 
3.2. Estimates for rings. Next we state results for rings analogous to those previously presented for paths. We omit the proofs since they are similar.

Lemma 3.7. Let $\alpha>1$ and $f: \mathbb{R} \rightarrow \mathbb{R}$ be defined by $f(x):=(2-2 \cos (2 \pi x))^{\alpha}$. Then

$$
\lim _{n \rightarrow \infty} n\left(\sum_{k=1}^{n} f\left(\frac{k}{n}\right)-n \frac{4^{\alpha} \Gamma(1 / 2+\alpha)}{\sqrt{\pi} \Gamma(1+\alpha)}\right)=0 .
$$

Proof. The proof is similar to the one of Lemma 3.3.

Notice that

$$
H_{\alpha}\left(C_{n}\right)=\frac{1}{1-\alpha}\left(\log \left(n \frac{4^{\alpha} \Gamma(1 / 2+\alpha)}{\sqrt{\pi} \Gamma(1+\alpha)}+R_{n}\right)-\alpha \log (2 n)\right) .
$$

We use the notation

$$
\widetilde{H}_{\alpha}\left(C_{n}\right)=\frac{1}{1-\alpha}\left(\log \left(n \frac{4^{\alpha} \Gamma(1 / 2+\alpha)}{\sqrt{\pi} \Gamma(1+\alpha)}\right)-\alpha \log (2 n)\right) .
$$

THEOREM 3.8. For $\alpha>1$,

$$
\lim _{n \rightarrow \infty} n\left(H_{\alpha}\left(C_{n}\right)-\widetilde{H}_{\alpha}\left(C_{n}\right)\right)=0,
$$

with $H_{\alpha}\left(C_{n}\right)$ and $\widetilde{H}_{\alpha}\left(C_{n}\right)$ in (3.8) and (3.9), respectively. Moreover, the approximation for integer values of $\alpha$ becomes exact, and

$$
\lim _{n \rightarrow \infty} \frac{H_{\alpha}\left(C_{n}\right)}{\log (n-1)}=1 .
$$

Proof. The proof is similar to those of Theorems 3.5 and 3.6.

In Table 3.5 we compare, $H_{3 / 2}\left(C_{n}\right), \widetilde{H}_{3 / 2}\left(C_{n}\right)$, and $H_{3 / 2}\left(K_{1, n-1}\right)$ using (3.8), (3.9), and (1.5). The last column suggests that $n R_{n}$ behaves like $1 / n^{2}$ so that $R_{n}$ behaves like $1 / n^{3}$.

\begin{tabular}{rccll}
\multicolumn{6}{c}{ Comparing $H_{3 / 2}\left(C_{n}\right), \widetilde{H}_{3 / 2}\left(C_{n}\right), H_{3 / 2}\left(K_{1, n-1}\right)$, and $n R_{n}}$. \\
$n$ & $H_{3 / 2}\left(C_{n}\right)$ & $\widetilde{H}_{3 / 2}\left(C_{n}\right)$ & $H_{3 / 2}\left(K_{1, n-1}\right)$ & \multicolumn{1}{c}{$n R_{n}$} \\
\hline 5 & 1.23979786 & 1.24409200 & 0.934611644 & 0.182445 \\
10 & 1.93698982 & 1.93723918 & 1.31230703 & 0.0423355 \\
20 & 2.63037105 & 2.63038636 & 1.55884276 & 0.0103965 \\
40 & 3.32353259 & 3.32353354 & 1.72360220 & 0.00258766 \\
80 & 4.01668066 & 4.01668072 & 1.83477278 & 0.000646201 \\
160 & 4.70982790 & 4.70982790 & 1.91029025 & 0.000161506
\end{tabular}

4. Estimating the von Neumann entropy of paths and rings. In this section we apply the E-M formula to the evaluation of the von Neumann entropy of the path $P_{n}$ for arbitrary $n$.

Let $f: \mathbb{R} \rightarrow \mathbb{R}$,

$$
f(x):=(2+2 \cos (\pi x)) \log (2+2 \cos (\pi x)) .
$$

Then (3.1) and (3.2) hold. Since $f(x)$ is not of class $C^{2}$ in $[0,1]$, the upper bound for $R_{n}$ in (1.2) is not useful. However, it holds that

$$
\lim _{n \rightarrow \infty} R_{n}=0 .
$$


Indeed, we clearly have

$$
\left|R_{n}\right| \leq \frac{1}{12 n} \int_{0}^{1}\left|f^{\prime \prime}(x)\right| \mathrm{d} x=\frac{2.41988}{n}
$$

since, numerically, we find

$$
\int_{0}^{1}\left|f^{\prime \prime}(x)\right| \mathrm{d} x=29.0386 .
$$

We estimate $R_{n}$. For this purpose, we consider the integral in (3.2)

$$
\frac{1}{n} \int_{0}^{1} B_{2}(\{n x\}) f^{\prime \prime}(t x) \mathrm{d} x=\int_{0}^{n} B_{2}(\{t\}) \frac{\mathrm{d}^{2} f(t / n)}{\mathrm{d} t^{2}} \mathrm{~d} t .
$$

As $f(x)$ is not of class $C^{2}$ in $[0,1]$, we consider the form (1.1) of the E-M formula. In order to deal with a divergence which arises in the integral, we split the integral over $t$ into two parts:

$$
\int_{0}^{n} B_{2}(\{t\}) \frac{\mathrm{d}^{2} f(t / n)}{\mathrm{d} t^{2}} \mathrm{~d} t=\int_{0}^{n-1} B_{2}(\{t\}) \frac{\mathrm{d}^{2} f(t / n)}{\mathrm{d} t^{2}} \mathrm{~d} t+\int_{n-1}^{n} B_{2}(\{t\}) \frac{d^{2} f(t / n)}{\mathrm{d} t^{2}} \mathrm{~d} t .
$$

By the version (1.3) of the E-M formula, the sum $\sum_{k=0}^{n-1} i(k)$ can be estimated by the integral $\int_{0}^{n} i(t) \mathrm{d} t$. To evaluate this integral, we consider

$$
\int_{0}^{n-1} B_{2}(\{t\}) \frac{\mathrm{d}^{2} f(t / n)}{\mathrm{d} t^{2}} \mathrm{~d} t .
$$

The fourth derivative of $f(t / n)$ is given by

$$
\frac{\mathrm{d}^{4} f}{\mathrm{~d} t^{4}}=\frac{\pi^{4}}{n^{4}}\left(-2+2 \cos \left(\frac{\pi t}{n}\right)\left(4+\log 2+\log \left(1+\cos \left(\frac{\pi t}{n}\right)\right)\right)-\sec ^{2}\left(\frac{\pi t}{2 n}\right)\right)=: g(t) .
$$

Thus,

$$
\begin{gathered}
i(k):=\int_{k}^{k+1} B_{2}(\{t\}) \frac{d^{2} f(t / n)}{\mathrm{d} t^{2}} \mathrm{~d} t=\frac{1}{360} g(k)+\ldots \\
=\frac{\pi^{4}}{360 n^{4}}(-2+2 \cos (\pi k / n)(4+\log 2+\log (1+\cos (\pi k / n))) \\
\left.\quad-\sec ^{2}(\pi k /(2 n))\right)+O(1 / n)
\end{gathered}
$$

where $O(1 / n)$ approaches 0 as $n \rightarrow \infty$.

By changing variables $x=k / n$ in the integral we find that

$$
\begin{aligned}
& \int_{0}^{n-1} i(k) \mathrm{d} k=n \int_{0}^{(n-1) / n} i(n x) \mathrm{d} x \\
& =\frac{1}{360} \frac{1}{n^{3}} \int_{0}^{1-1 / n} \pi^{3}\left(-2+2 \cos (\pi x)(4+\log 2+\log (1+\cos (\pi x)))-\sec ^{2}(\pi x / 2)\right) \mathrm{d} x \\
& =-\frac{4 \pi^{2}}{360 n^{2}}
\end{aligned}
$$

if $n$ is sufficiently large. 
Next we evaluate

$$
\int_{n-1}^{n} B_{2}(\{t\}) \frac{\mathrm{d}^{2} f(t / n)}{\mathrm{d} t^{2}} \mathrm{~d} t
$$

using

$$
\frac{\mathrm{d}^{2} f}{\mathrm{~d} k^{2}}=6 \pi^{2} n^{-2}+2 \pi^{2} n^{-2} \log \left(\left(\pi-\frac{k \pi}{n}\right)^{2}\right)+n^{-2} O(1 / n),
$$

which is valid for $n$ large and $k$ close to $n$. Hence, for $n$ large

$$
\int_{n-1}^{n} B_{2}(\{t\}) \frac{\mathrm{d}^{2} f(t / n)}{\mathrm{d} t^{2}} \mathrm{~d} t=-\frac{\pi^{2}}{9 n^{2}}+n^{-2} O(1 / n)
$$

and

$$
R_{n}=\frac{2 \pi^{2}}{360 n^{2}}+\frac{\pi^{2}}{18 n^{2}}+O\left(1 / n^{3}\right)=\frac{11 \pi^{2}}{180 n^{2}}+O\left(1 / n^{3}\right) .
$$

Thus $\lim _{n \rightarrow \infty} n R_{n}=0$, and so

$$
\lim _{n \rightarrow \infty} n\left(\sum_{k=1}^{n} f\left(\frac{k}{n}\right)-2 n+2 \log 4\right)=0 .
$$

The entropy of the path $P_{n}$ is given by

$$
S\left(P_{n}\right)=\log (2 n-2)-\frac{1}{2 n-2} \sum_{i=1}^{n} \beta_{i} \log \beta_{i},
$$

where $\beta_{1}, \ldots, \beta_{n}$ are the eigenvalues of $L\left(P_{n}\right)$ in (2.1). By the formula of Euler-Maclaurin, we may write as in the Lemma 3.1

$$
S\left(P_{n}\right)=\log (2 n-2)-\frac{1}{2 n-2}\left(2 n-4 \log 2+R_{n}\right) .
$$

We have

$$
S\left(P_{n}\right)-\widetilde{S}\left(P_{n}\right)=-\frac{1}{2 n-2} R_{n}
$$

where

$$
\widetilde{S}\left(P_{n}\right)=\log (2 n-2)-\frac{1}{2 n-2}(2 n-4 \log 2) .
$$

Now,

$$
\lim _{n \rightarrow \infty} n\left(S\left(P_{n}\right)-\widetilde{S}\left(P_{n}\right)\right)=0
$$

and further, since

$$
\frac{S\left(P_{n}\right)}{\log (n-1)}=1+\frac{1}{\log (n-1)}\left(\log (2)-\frac{1}{2 n-2}\left(2 n-4 \log 2+R_{n}\right)\right),
$$




\section{ETNA}

Kent State University and

Johann Radon Institute (RICAM)

ENTROPIES OF GRAPHS

TABLE 4.1

Comparing $S\left(P_{n}\right), \widetilde{S}\left(P_{n}\right), S\left(K_{1, n-1}\right)$, and $n R_{n}$.

\begin{tabular}{rcccl}
\multicolumn{1}{c}{$n$} & $S\left(P_{n}\right)$ & $\widetilde{S}\left(P_{n}\right)$ & $S\left(K_{1, n-1}\right)$ & \multicolumn{1}{c}{$n R_{n}$} \\
\hline 5 & 1.17298381 & 1.17601513 & 1.07354285 & 0.121253 \\
10 & 1.93295873 & 1.93329335 & 1.61115782 & 0.0602328 \\
20 & 2.65787788 & 2.65791744 & 2.06088496 & 0.0300676 \\
40 & 3.36660899 & 3.36661381 & 2.46497577 & 0.0150277 \\
80 & 4.06748424 & 4.06748484 & 2.84384737 & 0.00751311 \\
160 & 4.76448083 & 4.76448091 & 3.20850481 & 0.00375646
\end{tabular}

it follows that

$$
\lim _{n \rightarrow \infty} \frac{S\left(P_{n}\right)}{\log (n-1)}=1 .
$$

In Table 4.1 we compare the von Neumann entropy of the path $P_{n}$ with the approximate result denoted by $\widetilde{S}\left(P_{n}\right)$ and with the von Neumann entropy of $K_{1, n-1}$ using equations (4.1), (4.2), and (1.4). The values of $n R_{n}$ are also presented, indicating that $n R_{n}$ behaves like $1 / n$ so that $R_{n}$ behaves like $1 / n^{2}$. We notice that $\widetilde{S}\left(P_{n}\right)$ approaches $S\left(P_{n}\right)$ from above.

We next obtain similar results for the ring $C_{n}$ with $n$ vertices. Consider the function $f: \mathbb{R} \rightarrow \mathbb{R}, f(x):=(2-2 \cos (2 \pi x)) \log (2-2 \cos (2 \pi x))$, observe that $f(1)-f(0)=0$, $f^{\prime}(1)-f^{\prime}(0)=0$, and that

$$
\int_{0}^{1} f(x) \log f(x) \mathrm{d} x=2,
$$

as given by Mathematica. By Lemma 3.1, we obtain

$$
S\left(C_{n}\right)=\log (2 n)-\frac{1}{2 n}\left(2 n+R_{n}\right) .
$$

As $\lim _{n \rightarrow \infty} n R_{n}=0$, we have

$$
\lim _{n \rightarrow \infty}\left(S\left(C_{n}\right)-\widetilde{S}\left(C_{n}\right)\right)=0,
$$

where

$$
\widetilde{S}\left(C_{n}\right)=\log (2 n)-1
$$

Moreover,

$$
\lim _{n \rightarrow \infty} \frac{S\left(C_{n}\right)}{\log (n-1)}=1 .
$$

In Table 4.2 we compare the von Neumann entropy of the ring $C_{n}$ with the approximation $\widetilde{S}\left(C_{n}\right)$ and with the von Neumann entropy of $K_{1, n-1}$ using (4.3), (4.4), and (1.4). The last column suggests that $n R_{n}$ behaves like $1 / n$ so that $R_{n}$ behaves like $1 / n^{2}$.

5. Final remarks. In this note we have illustrated applications of the Euler-Maclaurin formula to the estimation of graph entropies of paths and rings. More generally, E-M formulas are available and potentially can be used in the same way as it was done for entropies of other graphs. From the previous Theorems 3.6 and 3.8 we conclude that, asymptotically, $H_{\alpha}\left(P_{n}\right)$ and $H_{\alpha}\left(C_{n}\right)$ behave as $\log (n-1)$. 
TABLE 4.2

Comparing $S\left(C_{n}\right), \widetilde{S}\left(C_{n}\right), S\left(K_{1, n-1}\right)$, and $n R_{n}$.

\begin{tabular}{rcccl}
\multicolumn{1}{r}{$n$} & $S\left(C_{n}\right)$ & $\widetilde{S}\left(C_{n}\right)$ & $S\left(K_{1, n-1}\right)$ & \multicolumn{1}{c}{$n R_{n}$} \\
\hline 5 & 1.28266167 & 1.30258509 & 1.07354285 & 0.996171 \\
10 & 1.99330722 & 1.99573227 & 1.61115782 & 0.485011 \\
20 & 2.68857829 & 2.68887945 & 2.06088496 & 0.240931 \\
40 & 3.38198905 & 3.38202663 & 2.46497577 & 0.120271 \\
80 & 4.07516912 & 4.07517382 & 2.84384737 & 0.0601109 \\
160 & 4.76832041 & 4.76832100 & 3.20850481 & 0.0300524
\end{tabular}

In Figure 5.1 we present $H_{\alpha}\left(P_{n}\right)-\widetilde{H}_{\alpha}\left(P_{n}\right)$ on the left-hand side and $R_{n}$ on the righthand side for $2 \leq n \leq 160$ and for $\alpha=1 / 2,3 / 2,5 / 2$ and $7 / 2$. It may be seen that $R_{n}$ and $H_{\alpha}\left(P_{n}\right)-\widetilde{H}_{\alpha}\left(P_{n}\right)$ decrease extremely fast in absolute value as $n$ increases. Indeed, these quantities are practically equal to 0 for $n>30$.
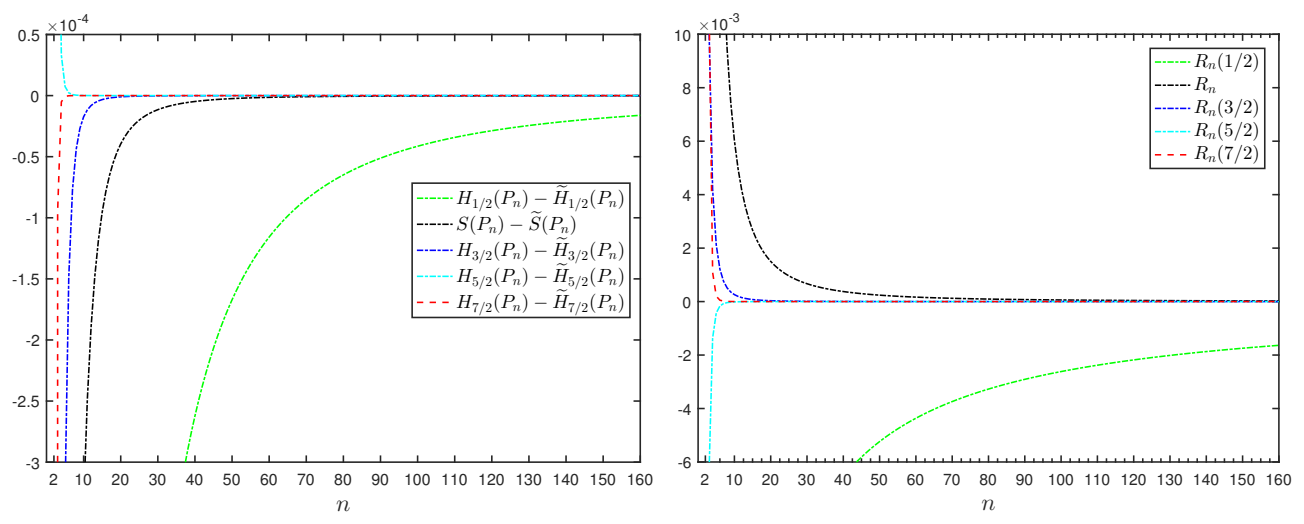

FIG. 5.1. The behavior of $H_{\alpha}\left(P_{n}\right)-\widetilde{H}_{\alpha}\left(P_{n}\right)$ on the left-hand side and of the corresponding $R_{n}$ on the right-hand side as $n$ increases. The curve labeled $R_{n}$ in the figure refers to the error in the sum $\sum_{k=1}^{n} \beta_{k} \log \beta_{k}$, where $\beta_{k}$ are the eigenvalues of (2.1).

In Figure 5.3, we present the von Neumann entropy $\widetilde{S}\left(P_{n}\right)$ for the path $P_{n}$ and the Rényi- $\alpha$ entropies $\widetilde{H}_{\alpha}\left(P_{n}\right)$ for $\alpha=\frac{1}{4}, \frac{1}{2}, \frac{3}{2}, 2$. We can see from this figure that

$$
\widetilde{H}_{1 / 4}\left(P_{n}\right)>\widetilde{H}_{1 / 2}\left(P_{n}\right)>\widetilde{S}\left(P_{n}\right)>\widetilde{H}_{3 / 2}\left(P_{n}\right)>\widetilde{H}_{2}\left(P_{n}\right)
$$

as expected. These entropies increase with $n$ for $n \geq 2$.

Acknowledgments. This work was partially supported by the Centro de Matemática da Universidade de Coimbra (CMUC), funded by the European Regional Development Fund through the program COMPETE and by the Portuguese Government through the Fundação para a Ciência e a Tecnologia (FCT) under the project PEst-C/MAT/UI0324/2011 and by CEAFEL under project UID/MAT/04721/2O3.

\section{REFERENCES}

[1] T. M. Apostol, An elementary view of Euler's summation formula, Amer. Math. Monthly, 106 (1999), pp. 409-418. 

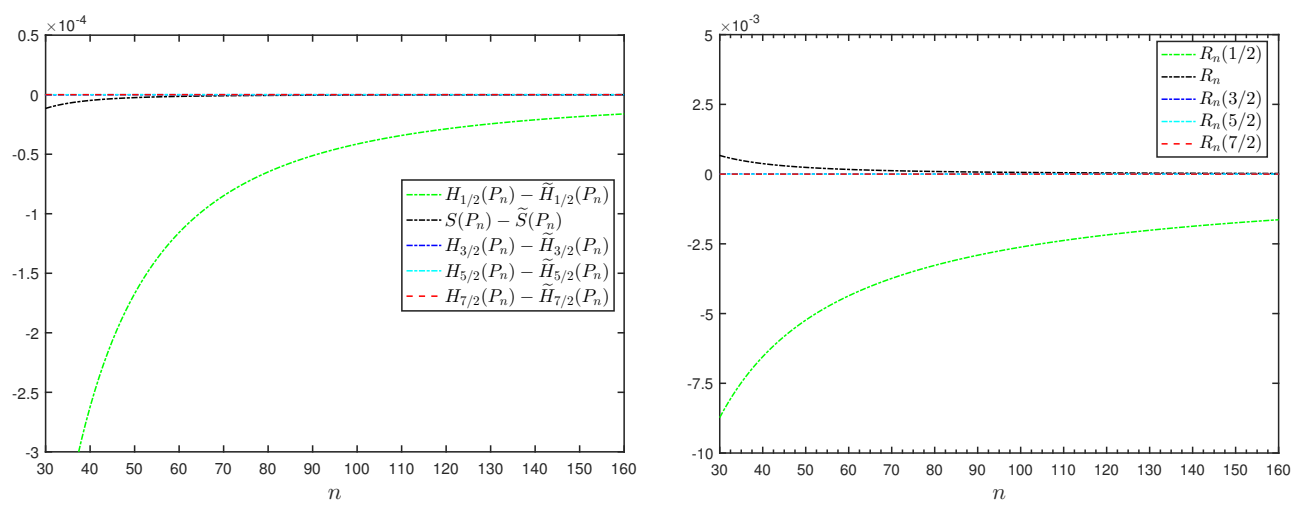

FIG. 5.2. Enlarged detail of Figure 5.1.
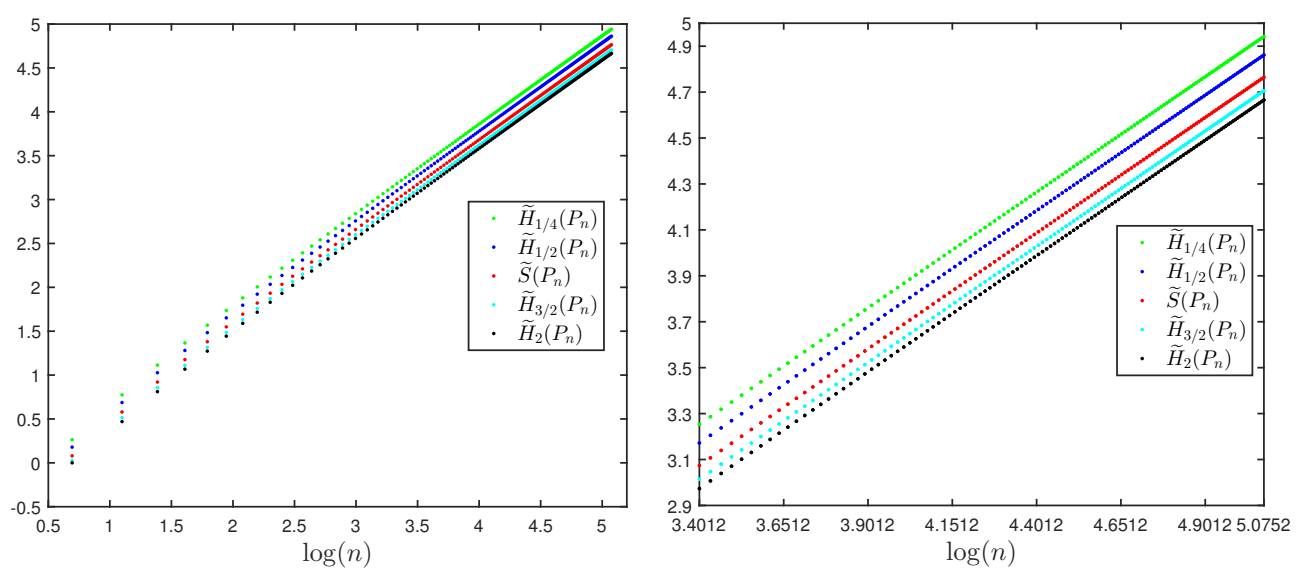

FIG. 5.3. The Rényi- $\alpha$ entropies of the path $P_{n}$ for $\alpha=\frac{1}{4}, \frac{1}{2}, \frac{3}{2}, 2$ and the entropy of the path $P_{n}$.

[2] D. Callan, A combinatorial survey of identities for the double factorial, Preprint on arXiv, 2009. https://arxiv.org/abs/0906.1317

[3] M. Dairyko, L. Hogben, J. C-H. Lin, J. Lockhart, D. Roberson, S. Severini, And M. Young, Note on von Neumann and Rényi entropies of a graph, Linear Algebra Appl., 521 (2017), pp. 240-253.

[4] V. GiovannetTi AND S. SEVERINI, The Kirchhoff's matrix tree theorem revisited: counting spanning trees with the quantum relative entropy, Preprint on arXiv, 2011.

https://arxiv.org/abs/1102.2398

[5] R. L. Graham, D. E. KnUth, And O. Patashnik, Concrete Mathematics: A Foundation for Computer Science, 2nd ed., Addison-Wesley, Reading, 1994.

[6] R. Grone, R. MERRIS, AND V. S. Sunder, The Laplacian spectrum of a graph, SIAM J. Matrix Anal. Appl., 11 (2017), pp. 218-238.

[7] R. A. Horn And C. R. Johnson, Matrix Analysis, 2nd ed., Cambridge University Press, Cambridge, 2013.

[8] V. LAMPRET, The Euler-Maclaurin and Taylor formulas: twin, elementary derivations, Math. Mag., 74 (2001), pp. 109-122.

[9] — , The Euler-Maclaurin formula and sums of powers revisited, Int. J. Contemp. Math. Sci., 5 (2010), pp. 2401-2407.

[10] J. von Neumann, Mathematical Foundations of Quantum Mechanics, Princeton University Press, Princeton, 1955.

[11] A. RÉNYI, On measures of entropy and information, in 1961 Proc. 4th Berkeley Sympos. Math. Statist. and Prob. Vol. I, J. Neyman, ed., Univ. California Press, Berkeley, 1961, pp. 547-561. 
[12] D. E. Simmons, J. P. Coon, And A. DatTA, Symmetric Laplacians, quantum density matrices and their von-Neumann entropy, Linear Algebra Appl., 532 (2017), pp. 534-549.

[13] M. Z. SPIVEY, The Euler-Maclaurin formula and sums of powers, Math. Mag., 79 (2006), pp. 61-65.

[14] S. Wolfram, The Mathematica Book, 4th ed., Cambridge University Press, Cambridge, 1999.

[15] C. Ye, R. C. Wilson, C. H. Comin, L. DA F. Costa, And E. R. Hanocock, Approximate von Neumann entropy for the direct graphs, Phys. Rev. E, 89 (2014), Art. 052804 (12 pages). 